\title{
miR-192-5p upregulation mediates the suppression of curcumin in human NSCLC cell proliferation, migration and invasion by targeting c-Myc and inactivating the Wnt/ $\beta$-catenin signaling pathway
}

\author{
YANCHENG PAN $^{1 *}$, YING SUN $^{2 *}$, ZIQI LIU $^{2}$ and CHAO ZHANG ${ }^{1}$ \\ ${ }^{1}$ Department of Pharmacy, The Tengzhou Central People's Hospital, Tengzhou, Shandong 277500; \\ ${ }^{2}$ Department of Pharmacy, Yantai Hospital of Traditional Chinese Medicine, Yantai, Shandong 264000, P.R. China
}

Received August 1, 2019; Accepted January 30, 2020

DOI: $10.3892 / \mathrm{mmr} .2020 .11213$

\begin{abstract}
Curcumin is a naturally active phenolic compound extracted from the rhizome of the plant Curcuma longa, which has been demonstrated to serve as an anticancer drug in different types of cancer, including non-small-cell lung cancer (NSCLC). Accumulating evidence has suggested that curcumin may exert epigenetic regulatory effects on microRNAs (miRs). Therefore, the present study aimed to investigate the role of miR-192-5p, and the effects of curcumin, in NSCLC, alongside the underlying mechanisms. Human NSCLC cells, A427 and A549, were treated with curcumin, and the expression levels of miR-192-5p and c-Myc were detected using reverse transcription-quantitative PCR and western blotting. Cellular proliferation was analyzed using Cell Counting Kit-8 assays and cell viability was determined using a MTT assay. Additionally, the migratory and invasive abilities of cells were analyzed using Transwell and Matrigel assays, respectively. The binding sites between miR-192-5p and c-Myc were predicted using TargetScanHuman software, and confirmed using a dual-luciferase reporter assay and RNA immunoprecipitation. Finally, the Wnt pathway regulator, $\beta$-catenin, and cyclin D1 expression levels were determined using western blotting. Curcumin treatment inhibited NSCLC cell proliferation, migration, invasion and viability in a dose-dependent manner, in addition to promoting a dose-dependent increase in the expression levels of miR-192-5p and a reduction in c-Myc expression levels. Notably, the genetic knockdown of miR-192-5p blocked the inhibitory effects of curcumin on
\end{abstract}

Correspondence to: Mr. Chao Zhang, Department of Pharmacy, The Tengzhou Central People's Hospital, 181 Xingtan Road, Tengzhou, Shandong 277500, P.R. China

E-mail: teoojw@163.com

*Contributed equally

Key words: microRNA-192-5p, curcumin, non-small-cell lung cancer, c-Myc, Wnt/ß-catenin pathway
NSCLC progression and instead promoted NSCLC progression, which was observed to be partially reversed by c-Myc silencing; thus, c-Myc was suggested to be a direct target gene of miR-192-5p as demonstrated by the TargetScanHuman database, dual-lucierase and RIP assay results. In addition, the curcumin-induced decreased expression levels of $\beta$-catenin, cyclin D1 and c-Myc were rescued following the genetic knockdown of miR-192-5p. In conclusion, these findings suggested that the upregulation of miR-192-5p may underlie the inhibitory effects of curcumin on NSCLC cells through targeting c-Myc and inactivating the Wnt/ $\beta$-catenin signaling pathway.

\section{Introduction}

Non-small-cell lung cancer (NSCLC) is one of the most fatal cancer types worldwide, accounting for $\sim 80 \%$ of all primary lung cancer types (1). Statistically, 70-80\% of patients with NSCLC will progress to an advanced disease state once diagnosed (2). With regards to the current treatment options, intensive chemotherapy combined with aggressive surgical techniques have demonstrated significant successes; however, the prognosis of patients with NSCLC remains disappointing due to the chemoresistance, invasion and metastasis of the tumors $(3,4)$. Thus, there is an urgent requirement to develop novel chemotherapies for the treatment of NSCLC.

Curcumin is a naturally active phenolic compound extracted from the rhizome of the plant Curcuma longa, which is used as a traditional Chinese medicine (5). Curcumin has become an increasingly popular compound of interest, both in research and clinically, among different types of cancer, including lung adenocarcinoma, a type of NSCLC (6-8). It is reported to have anticancer properties that are exerted by affecting multiple cellular processes, such as cell proliferation, invasion, metastasis and chemoresistance (9); however, the mechanisms of action of curcumin in NSCLC remain largely unknown.

MicroRNAs (miRNAs/miRs) are a class of endogenous small non-coding RNAs of $\sim 22$ nucleotides in length that interact with mRNAs, which leads to mRNA degradation or the inhibition of translation (10). Functionally, miRNAs 
are involved in diverse cellular functions, such as growth, proliferation, apoptosis and migration (11). Accumulating evidence has demonstrated that curcumin may represent a novel strategy for cancer treatment by serving as an epigenetic regulator; initially, curcumin was identified to exert epigenetic activity over miRNAs in pancreatic cancer (12), and since this, other studies have also reported an association between the pharmacological effect of curcumin and miRNAs in lung cancer (10). For example, Ye et al (13) discovered that miR-192-5p and miR-215 were involved in the proapoptotic effects of curcumin, whereas Jin et al (14) also suggested that the antiproliferative role of curcumin in human NSCLCs depended on the increased expression levels of miR-192-5p and the inactivation of the PI3K/AKT signaling pathway. However, apart from these studies, to the best of our knowledge, there are very few studies into the role of miR-192-5p in NSCLC progression, especially in processes such as migration and invasion. In addition, the underlying regulatory mechanism of curcumin in NSCLC remains to be fully investigated.

The Wnt/ $\beta$-catenin signaling pathway is one of the main and most frequently dysregulated pathways in several types of tumor, and it is suggested that altered cell proliferation, invasive behaviors and cell resistance are all attributed to the dysregulation of this signaling pathway $(15,16)$. Notably, several studies have reported that curcumin affects cell proliferation through the Wnt/ $\beta$-catenin signaling pathway in multiple different types of cancer, including both colon cancer and medulloblastoma $(17,18)$. In fact, in lung cancer, this signaling pathway has been demonstrated to participate in the anticancer role of curcumin in tumor cell growth, invasion, epithelial-mesenchymal transition and cancer stem cells traits $(15,16,19,20)$. In addition, the involvement of miR-192-5p and the $\mathrm{Wnt} / \beta$-catenin signaling pathway is noted in several types of tumor (21-23), excluding lung cancer; however, little is known regarding the interaction between curcumin, miR-192-5p and the Wnt/ $\beta$-catenin signaling pathway in NSCLC.

In the present study, the expression levels of miR-192-5p and c-Myc, an important oncogene, were investigated in NSCLC cells following curcumin treatment. In addition, functional and mechanistic assays were used to investigate the effect of miR-192-5p dysregulation on curcumin-mediated cell proliferation, invasion, migration and the $\mathrm{Wnt} / \beta$-catenin signaling pathway in NSCLC cells. In short, this study suggested a novel molecular mechanism for curcumin in NSCLC.

\section{Materials and methods}

Cell culture and reagents. The human NSCLC cell lines, A427 and A549, and the human embryonic kidney cell line 293T were purchased from the American Type Culture Collection. The NSCLC cells were cultured in RPMI-1640 medium (HyClone; GE Healthcare Life Sciences) and 293T cells were maintained in DMEM (HyClone; GE Healthcare Life Sciences). Both mediums were supplemented with $10 \%$ FBS (HyClone; GE Healthcare Life Sciences) and 1\% penicillin/streptomycin (Invitrogen; Thermo Fisher Scientific, Inc.) and cells were then maintained in a humidified atmosphere of $5 \% \mathrm{CO}_{2}$ at $37^{\circ} \mathrm{C}$.
Curcumin treatment. Curcumin (100 mM in DMSO) was purchased from Sigma-Aldrich (Merck KGaA). The working concentration of curcumin was 10,20 or $40 \mu \mathrm{M}$ diluted in culture medium. Cells were cultured in 6-well or 96-well plates (Corning, Inc.) and exposed to $10-40 \mu \mathrm{M}$ curcumin for $48 \mathrm{~h}$ at $37^{\circ} \mathrm{C}$. The control group was treated with $0 \mu \mathrm{M}$ of curcumin diluted in $0.1 \%$ DMSO (24). The $20 \mu \mathrm{M}$ curcumin concentration was selected to treat A427 and A549 cells for further analysis.

Cell transfection. The hsa-miR-192-5p mimic (5'-CUGACC UAUGAAUUGACAGCC-3') and miR-negative control (NC) mimic (5'-UUCUCCGAACGUGUCACGUTT-3') were obtained from Shanghai GenePharma Co., Ltd. The pcDNA3.1 (pcDNA; Invitrogen; Thermo Fisher Scientific, Inc.) plasmid was used to construct the c-Myc overexpression vector (pcDNA-c-Myc). Small interfering (si)RNA against c-Myc (si-c-Myc; 5'-CCTGAGACAGATCAGCAACAA-3') and its NC (si-NC; 5'-TTCTCCGAACGTGTCACGT-3'), and the hsa-miR-192-5p inhibitor (in-miR-192-5p; 5'-GGC UGUCAAUUCAUAGGUCAG-3') and miR-NC inhibitor (in-miR-NC; 5'-CAGUACUUUUGUGUAGUACAA-3') were purchased from Shanghai GenePharma Co., Ltd. Cells at $70 \%$ confluency were cultured in 6-well plates (Corning, Inc.) and the transfection of plasmids $(2 \mu \mathrm{g})$ and oligonucleotides (40 nM) was performed using Lipofectamine ${ }^{\circledR} 2000$ reagent (Invitrogen; Thermo Fisher Scientific, Inc.), according to the manufacturer's protocol. Cells were transfected for $48 \mathrm{~h}$ at $37^{\circ} \mathrm{C}$ prior to further studies.

MTT staining for cell viability. Cell viability was determined using an MTT reagent (Sangon Biotech Co., Ltd.). Following curcumin treatment, $5 \times 10^{-3} \mathrm{mg} / \mathrm{ml}$ MTT was added to each well and incubated for $4 \mathrm{~h}$ at $37^{\circ} \mathrm{C}$. The culture medium containing MTT was discarded with micropipettor and formazan crystals were dissolved in $100 \mu$ l dimethyl sulfoxide. The optical density (OD) was measured at $490 \mathrm{~nm}$ using a SpectraMax M4 microplate reader (Molecular Devices, LLC). Cell viability (\%) was calculated using the following formula: OD value of treated group/OD value of control group x100. All experiments were performed in quadruplicate.

Cell Counting Kit-8 (CCK-8) assay for cell proliferation. The proliferative ability of the NSCLC cells was determined using a CCK-8 assay (Beyotime Institute of Biotechnology), according to the manufacturer's protocol. Briefly, $1 \times 10^{4}$ A427 and A549 cells/well were plated into 96-well plates (Corning, Inc.) for $24 \mathrm{~h}$ at $37^{\circ} \mathrm{C}$. Subsequently, $20 \mu \mathrm{l} \mathrm{CCK}-8$ solution $(5 \mathrm{mg} / \mathrm{ml})$ diluted in PBS was added to each well for another $1 \mathrm{~h}$ and the absorbance was measured at $450 \mathrm{~nm}$ using a SpectraMax M4 microplate reader (Molecular Devices, LLC). Results were expressed as a percentage relative to the control group (defined as $100 \%$ ). Data are from $\geq 5$ independent experiments.

Reverse transcription-quantitative PCR (RT-qPCR). Total RNA was extracted from cells using TRIzol ${ }^{\circledR}$ reagent (Invitrogen; Thermo Fisher Scientific, Inc.), according to the manufacturer's protocol. Total RNA was reverse transcribed into cDNA using a PrimeScript ${ }^{\mathrm{TM}}$ RT reagent kit (Takara Bio, Inc.) according to the manufacturer's instuctions. The 
temperature conditions were as followed: $25^{\circ} \mathrm{C}$ for $10 \mathrm{~min}$, $42^{\circ} \mathrm{C}$ for $15 \mathrm{~min}$ and $85^{\circ} \mathrm{C}$ for $5 \mathrm{~min}$. qPCR was subsequently performed using a SYBR Green PCR Master mix (Takara Bio, Inc.) and a TaqMan miRNA assay kit (Applied Biosystems; Thermo Fisher Scientific, Inc.) according to the manufacturer's protocol to amplify mRNAs and miRNAs, respectively, on an ABI PRISM 7500 Real-time PCR system (Applied Biosystems; Thermo Fisher Scientific, Inc.). The thermocycling conditions were as follows: Initial denaturation was $95^{\circ} \mathrm{C}$ for $5 \mathrm{~min}$, followed by 40 cycles of $95^{\circ} \mathrm{C}$ for $10 \mathrm{sec}$ and $60^{\circ} \mathrm{C}$ for $30 \mathrm{sec}$, and the melting curve generation condition was $95^{\circ} \mathrm{C}$ for $15 \mathrm{sec}, 60^{\circ} \mathrm{C}$ for $60 \mathrm{sec}$ and $95^{\circ} \mathrm{C}$ for $15 \mathrm{sec}$. The following primer pairs were used for the qPCR: c-Myc forward, 5'-GGC TCCTGGCAAAAGGTCA-3' and reverse, 5'-CTGCGTAGT TGTGCTGATGT-3'; miR-192-5p forward, 5'-GGACTTTCT TCATTCACACCG-3' and reverse, 5'-GACCACTGAGGT TAGAGCCA-3'; GAPDH forward, 5'-CGAGCCACATCG CTCAGACA-3' and reverse, 5'-GTGGTGAAGACGCCAGTG GA-3'; and U6 forward, 5'-TCGCTTCGGCAGCACATATAC T-3' and reverse, 5'-ACGCTTCACGAATTTGCGTGTC-3'. Expression levels were quantified using the $2^{-\Delta \Delta \mathrm{Cq}}$ method (25) and miRNA was normalized to U6 (miRNA) and GAPDH (mRNA). The reactions were performed in quadruplicate for each sample, with $\geq 3$ independent runs.

Western blotting. Total protein was extracted from cells using RIPA lysis buffer (Beyotime Institute of Biotechnology). Total protein was quantified with Bradford method and $20 \mu \mathrm{g}$ protein/lane was separated via 10-12\% SDS-PAGE, and then transferred onto PVDF membranes. After blocking with $5 \%$ skimmed milk for $2 \mathrm{~h}$ at $25^{\circ} \mathrm{C}$, the membranes were incubated with the following primary antibodies (all Abcam): Anti-c-Myc (1:1,000; cat. no. ab32072), anti- $\beta$-catenin $(1: 4,000$; cat. no. ab6302), anti-cyclin D1 (1:5,000; cat. no. ab40754) and anti- $\beta$-actin $\left(1: 5,000\right.$; cat.no. ab8227) for overnight at $4^{\circ} \mathrm{C}$. After incubation with horseradish peroxidase-conjugated secondary antibody anti-Rabbit (1:50,000; Abcam; cat. no. ab205718) for $1 \mathrm{~h}$ at $25^{\circ} \mathrm{C}$, the protein bands were visualized using an ECL reagent (EMD Millipore). Protein expression was normalized to the loading control $\beta$-actin on Image-Pro Plus 6.0 software (Media Cybernetics, Inc.).

Computational prediction. TargetScanHuman 7.1 database (http://www.targetscan.org/ENST00000377970.2) was used to predict the potential miRNAs targeting Myc gene on the 3'untranslated region (c-Myc 3'UTR). The in silico data provided a potential binding site between miR-192-5p and c-Myc 3'UTR on position 738-744.

Dual-luciferase reporter assay. The pMIR-REPORT Luciferase miRNA Expression Reporter Vector and pMIR-REPORT $\beta$-galactosidase Reporter Control Vector, were purchased from Invitrogen; Thermo Fisher Scientific, Inc. The putative binding site of miR-192-5p in c-Myc 3'UTR was mutated. The wild-type (WT) of c-Myc 3'UTR fragment (c-Myc-3'UTR-WT) and the mutant type (MUT) of c-Myc 3'UTR fragment (c-Myc-3'UTR-MUT) were cloned into the pMIR-REPORT miRNA Expression Reporter Vector, respectively. 293T cells at $60 \%$ confluency were co-transfected with 100 ng pMIR-REPORT-c-Myc-WT/MUT vectors, pMIR-REPORT $\beta$-galactosidase vectors and $50 \mathrm{nM}$ miR-192-5p mimic/NC mimic using Lipofectamine ${ }^{\circledR} 2000$ reagent (Invitrogen; Thermo Fisher Scientific, Inc.) according to the manufacturer's protocol. Following incubation for $48 \mathrm{~h}$ at $37^{\circ} \mathrm{C}$, transfected $293 \mathrm{~T}$ cells were collected and the firefly luciferase and $\beta$-galactosidase activities were detected using a Dual-Luciferase Reporter assay system (Promega Corporation) according to the manufacturer's protocol. Firefly luciferase activity was normalized to $\beta$-galactosidase expression levels. Data are presented as the mean of $\geq 3$ independent transfections.

RNA immunoprecipitation (RIP). Following the transfection of miR-192-5p mimic or miR-NC, RIP was performed in cell extracts in RIPA lysis buffer (Beyotime Institute of Biotechnology). A Magna RIP ${ }^{\mathrm{TM}}$ RNA-binding protein immunoprecipitation kit (EMD Millipore) was used according to the manufacturer's protocol to obtain the RIP that bound to argonaute 2 (Ago2; Abcam; 1:20; cat. no. ab32381) or IgG (1:50; Abcam; cat. no. ab2410) antibody. Then, RT-qPCR was performed to detect the expression levels of c-Myc mRNA in RIP.

Transwell assays. For the migration and invasion assays, A427 and A549 cells $\left(5 \times 10^{5}\right)$ were suspended in $200 \mu$ l RPMI-1640 medium without FBS and plated in the upper chambers of Transwell plates (Corning, Inc.) without or with Matrigel (Corning, Inc.) for migration and invasion, respectively. The Matrigel membrane was established using Matrigel (1:8) after air drying for $16 \mathrm{~h}$ at $4^{\circ} \mathrm{C}$. RPMI-1640 medium supplemented with $10 \%$ FBS (Gibco; Thermo Fisher Scientific, Inc.) was plated in the lower chambers. Following incubation at $37^{\circ} \mathrm{C}$ for $48 \mathrm{~h}$, the migratory and invasive cells in the lower chambers were stained with $0.2 \%$ crystal violet for $15 \mathrm{~min}$ at $25^{\circ} \mathrm{C}$. Absorbance was measured at a wavelength of $570 \mathrm{~nm}$ using a microplate reader and the migratory/invasive ability was presented as a percentage of the control group.

Statistical analysis. Statistical analysis was performed using GraphPad Prism 5.0 software (GraphPad Software, Inc.) and data are presented as the mean \pm SD. Statistical differences between groups were determined using a one-way ANOVA with Tukey's post hoc analysis. $\mathrm{P}<0.05$ was considered to indicate a statistically significant result.

\section{Results}

Curcumin treatment suppresses NSCLC cell progression in vitro. Following the exposure of NSCLC A427 and A549 cells to different concentrations of curcumin (10, 20 and $40 \mu \mathrm{M})$, the tumor-suppressive role of curcumin in NSCLC in vitro was investigated. Cellular proliferation was significantly decreased in both cell lines following 10, 20 and $40 \mu \mathrm{M}$ curcumin treatment compared with the control cells (Fig. 1A and B), which was consistent with the cell viability status (Fig. 1G and $\mathrm{H}$ ). In addition, the migratory ability of NSCLC cells was determined; the migratory ability of both cell lines was significantly attenuated by 10,20 and $40 \mu \mathrm{M}$ of curcumin compared with the control (Fig. 1C and D). Curcumin was also found to significantly reduce the invasive 
A

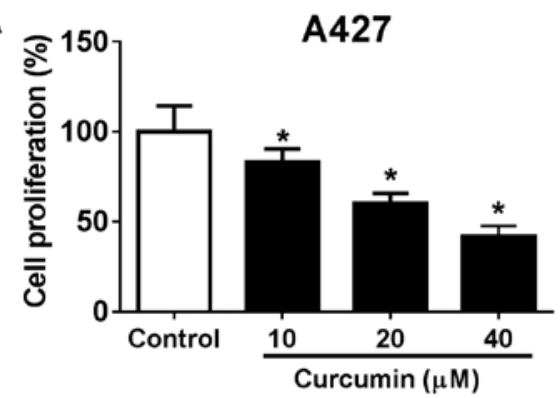

C

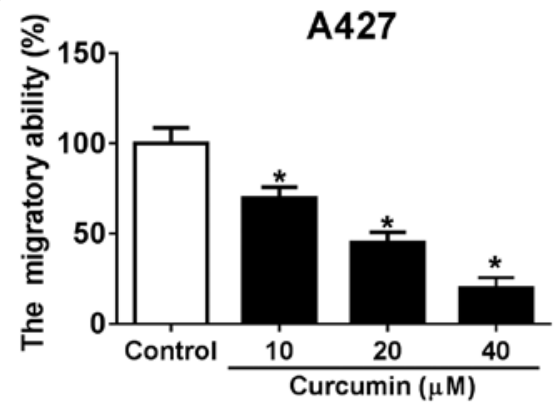

E

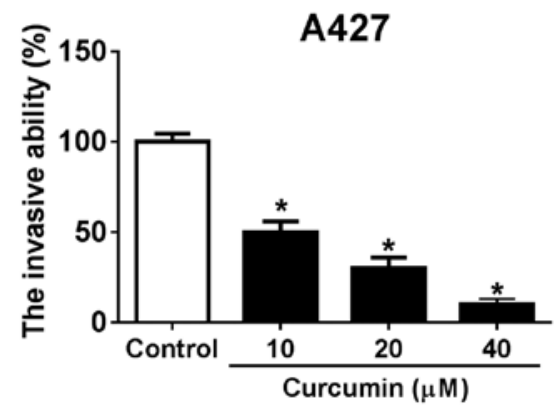

G

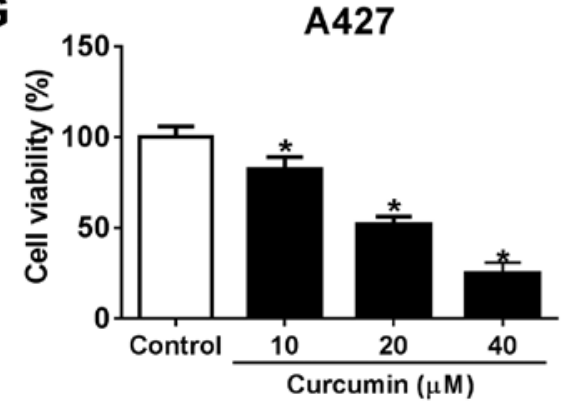

B
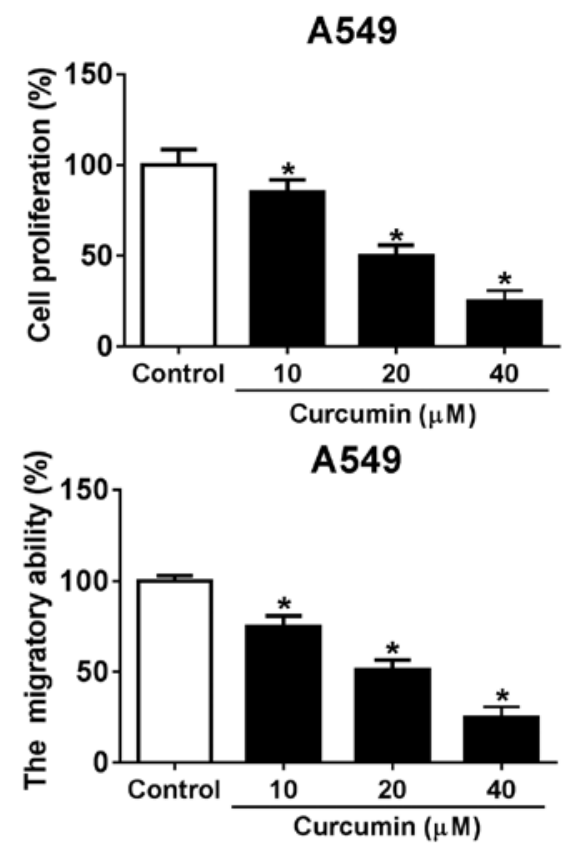

$\mathbf{F}$

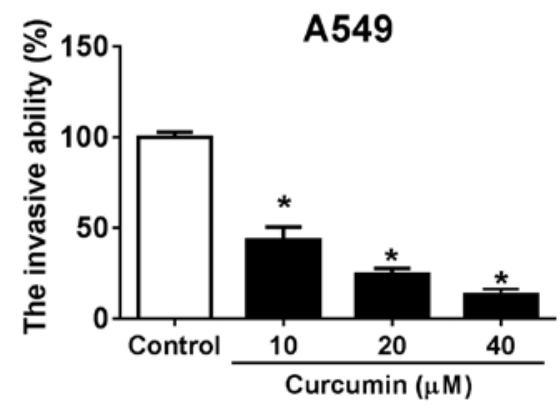

H

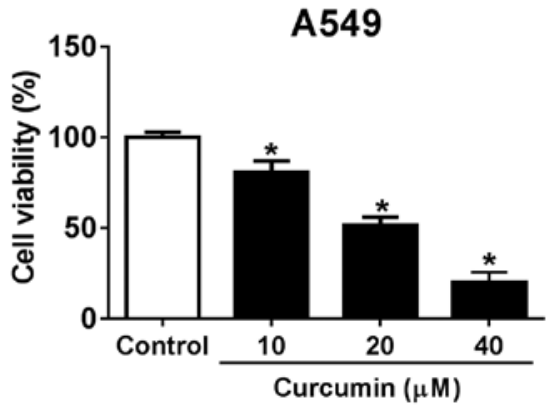

Figure 1. Effects of curcumin on non-small-cell lung cancer cells. A427 and A549 cells were treated with 10,20 or $40 \mu \mathrm{M}$ curcumin for 48 h. Cell proliferation rates in (A) A427 and (B) A549 cells were determined using a Cell Counting Kit-8 assay. Cell migration in (C) A427 and (D) A549 cells was determined using a Transwell assay. Cell invasion in (E) A427 and (F) A549 cells was determined using a Matrigel assay. Cell viability of (G) A427 and (H) A549 cells exposed to curcumin was measured using an MTT assay. ${ }^{*} \mathrm{P}<0.05$ vs. control $(0 \mu \mathrm{M})$.

ability of both cell lines at all concentrations (10, 20 and $40 \mu \mathrm{M}$ ) compared with the control (Fig. 1E and F). Of note, the effects of curcumin on NSCLCs were all observed to occur in a dose-dependent manner. These results suggested that curcumin treatment may promote NSCLC cell cytotoxicity and suppress cell migration and invasion, leading to the antitumor effect in NSCLC in vitro.

Expression levels of miR-192-5p and c-Myc are altered following curcumin treatment in NSCLC. To determine the potential association between miR-192-5p and c-Myc in NSCLC, RT-qPCR and western blotting were used to quantify their expression levels in both A427 and A549 cells exposed to different concentrations of curcumin (10, 20 and $40 \mu \mathrm{M})$. Curcumin treatment led to a significant concentration-dependent increase in miR-192-5p expression levels compared with the control (Fig. 2A and B), whereas the expression levels of c-Myc at both the mRNA (Fig. 2C and D) and protein level (Fig. 2E and F) were significantly decreased in a dose-dependent manner in both cell lines compared with the control. These findings suggested that miR-192-5p expression was increased and c-Myc expression was decreased in NSCLC cells following curcumin exposure, which may contribute to curcumin-induced NSCLC cell cytotoxicity and suppression of cell migration and invasion. Subsequently, the role of miR-192-5p and c-Myc in the role of curcumin in NSCLC 
A

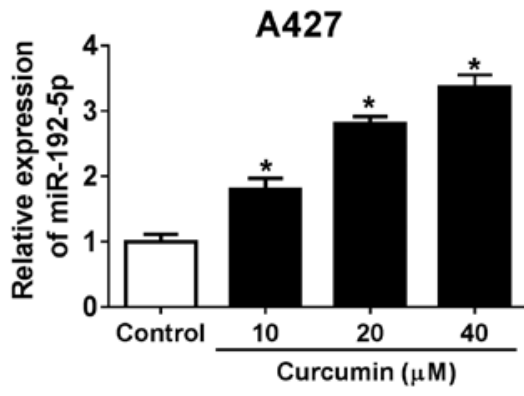

C

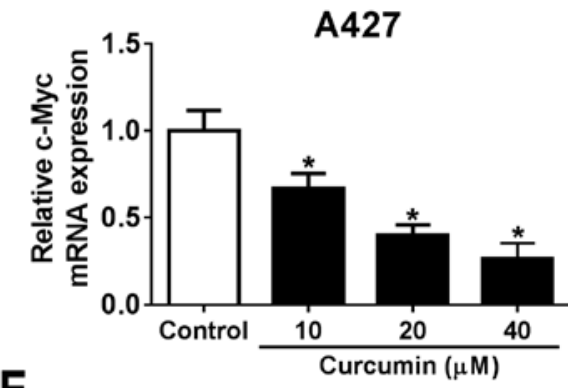

E
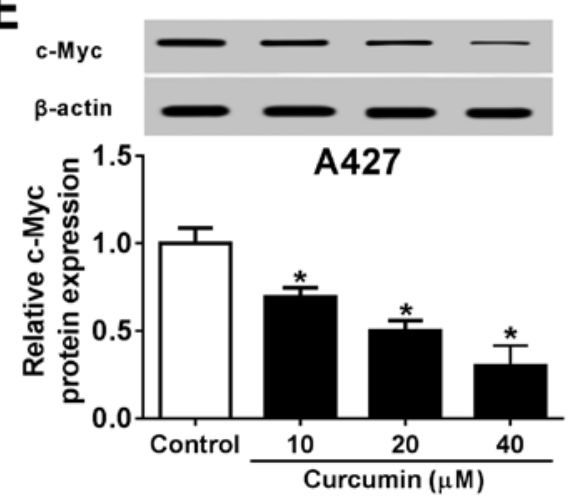

B

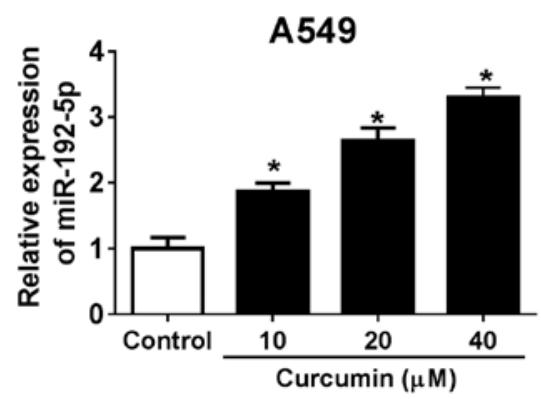

A549

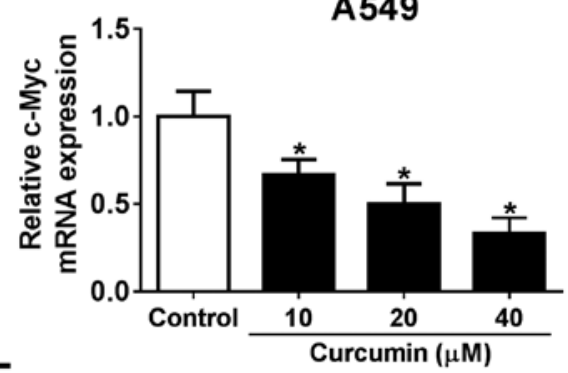

$\mathbf{F}$
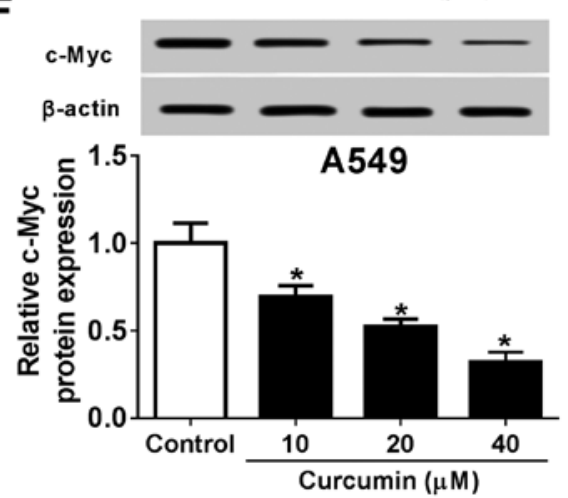

Figure 2. Effects of curcumin on the expression levels of miR-192-5p and c-Myc in non-small-cell lung cancer. A427 and A549 cells were treated with 10, 20 or $40 \mu \mathrm{M}$ curcumin for $48 \mathrm{~h}$. Effects of curcumin treatment on miR-192-5p expression levels in (A) A427 and (B) A549 cells were analyzed using RT-qPCR. Effects of curcumin treatment on c-Myc expression levels in (C) A427 and (D) A549 cells were analyzed using RT-qPCR. Effects of curcumin on c-Myc protein expression levels in (E) A427 and (F) A549 cells were determined using western blotting. ${ }^{*} \mathrm{P}<0.05$ vs. control (0 $\left.\mu \mathrm{M}\right)$. miR, microRNA; RT-qPCR, reverse transcription-quantitative PCR.

cells were further investigated, and $20 \mu \mathrm{M}$ of curcumin was used (Figs. 1 and 2).

miR-192-5p knockdown reverses the inhibitory effects of curcumin on NSCLC cell proliferation, migration and invasion. The dysregulation of miR-192-5p expression following curcumin treatment was confirmed in the human NSCLC cell lines, A427 and A549. Thus, the functions of miR-192-5p following its knockdown by transfection in curcumin-induced anticancer activity were further investigated. Following the transient transfection of the in-miR-192-5p into A427 and A549 cells, a significant decrease was observed in miR-192-5p expression levels in A427 and A549 cells compared with the in-miR-NC-transfected cells, as indicated by RT-qPCR (Fig. S1A). The increased miR-192-5p expression levels induced by curcumin were impaired following the exogenous administration of in-miR-192-5p (Fig. 3A). In addition, following $20 \mu \mathrm{M}$ curcumin treatment, in-miR-192-5p-transfected cells demonstrated significantly increased proliferation rates in A427 and A549 cells compared with the curcumin + in-miR-NC group (Fig. 3B). The curcumin induced a decrease in cell migratory and invasive abilities in A427 and A549 cells, which was significantly reversed by miR-192-5p inhibition (Fig. 3C and D). These results indicated that the beneficial effects of curcumin were partially abolished by miR-192-5p knockdown, suggesting that miR-192-5p upregulation may be behind the underlying anticancer role of curcumin in NSCLC.

$c-M y c$ is a direct target of miR-192-5p. Accumulating evidence has reported that c-Myc undertakes important roles in the numerous biological functions of miRNAs in NSCLC (26-28); however, the regulatory effect of miR-192-5p on c-Myc remains largely unknown. In this study, a potential binding site between miR-192-5p and c-Myc was identified using computational prediction on the TargetScanHuman database (Fig. 4A). Then, c-Myc-WT and c-Myc-MUT 3'UTRs were cloned into pMIR vectors, and a dual-luciferase reporter assay was used to demonstrate that the relative luciferase activity of c-Myc-3'UTR-WT in 293T cells was significantly reduced following co-transfection with miR-192-5p mimic compared with the miR-NC group (Fig. 4B), whereas the relative luciferase activity was significantly increased following co-transfection with in-miR-192-5p compared with the in-miR-NC group 
A

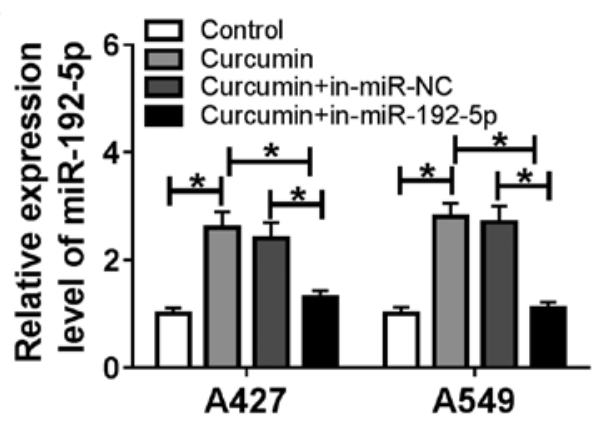

C

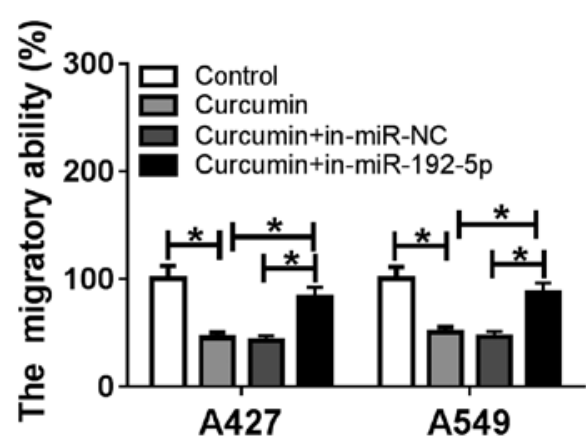

B

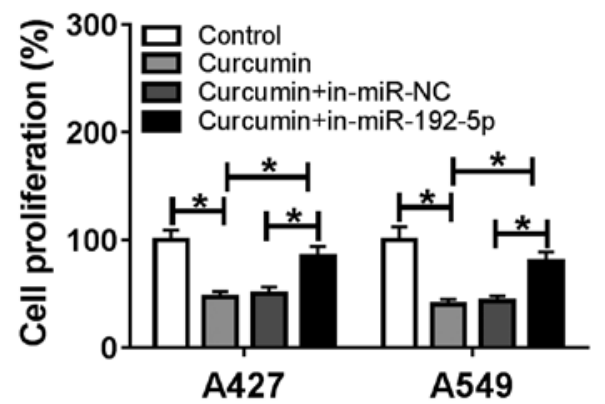

D

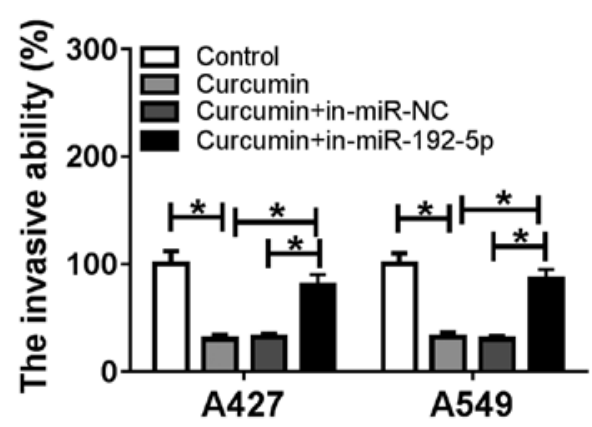

Figure 3. Influence of miR-192-5p knockdown on curcumin-induced NSCLC progression. A427 and A549 cells were transfected with in-miR-192-5p or miR-NC and the effect of the in-miR-192-5p on NSCLC cells treated with $20 \mu \mathrm{M}$ curcumin was investigated. (A) Expression levels of miR-192-5p in each group were detected in A427 and A549 cells using reverse transcription-quantitative PCR. (B) Cell proliferation of each group was determined in A427 and A549 cells using a Cell Counting Kit-8 assay. (C) Cell migratory ability in each group was determined in A427 and A549 cells using a Transwell assay. (D) Invasive ability in each group was determined in A427 and A549 cells using a Matrigel assay. *P<0.05. miR, microRNA; NSCLC, non-small-cell lung cancer; NC, negative control; in, inhibitor.

(Fig. 4C). The miR-192-5p mimic transfection was also validated; a significant increase in miR-192-5p expression levels was observed in A427 and A549 cells in the miR-192-5p mimic-transfected cells compared with the miR-NC group, as indicated by RT-qPCR (Fig. S1B). However, there were no significant differences in c-Myc-3'UTR-MUT-transfected cells between the NCs- and inhibitor/mimic-transfected cells (Fig. 4B and C). A RIP assay further validated the existence of a binding site between miR-192-5p and c-Myc (Fig. 4D), and western blotting demonstrated that c-Myc expression was significantly inhibited by the miR-192-5p mimic in A427 and A549 cells compared with the miR-NC group (Fig. 4E). These results suggested that miR-192-5p may negatively regulate c-Myc expression through target binding.

Curcumin exerts its anticancer role through the $\mathrm{miR}-192-5 \mathrm{p} / \mathrm{c}$ $M y c$ axis in NSCLC in vitro. Rescue experiments were used to clarify the role of c-Myc in mediating the biological action of miR-192-5p in A427 and A549 cells treated with curcumin. The transfection of in-miR-192-5p in both cell lines under curcumin treatment significantly increased the expression levels of c-Myc compared with the in-miR-NC group (Fig. 5A), whereas the co-transfection of in-miR-192-5p and si-c-Myc resulted in a significant decrease in c-Myc expression levels compared with the in-miR-192-5p + si-NC group. In addition, in cells transfected with in-miR-192-5p, cell proliferation was significantly increased compared with the in-miR-NC group (Fig. 5B); however, in combination with si-c-Myc, this increase was subsequently blocked (Fig. 5B). The downregulation of
c-Myc expression in in-miR-192-5p-transfected cells also partly abolished the promotive effects of in-miR-192-5p transfection on cell migration and invasion (Fig. 5C and D). The transfection efficiency of si-c-Myc was assessed using RT-qPCR and western blotting (Fig. S1C and D). Moreover, pcDNA-c-Myc resulted in the overexpression of c-Myc (Fig. S1E and F), and this transfection also counteracted the suppressive effect of miR-192-5p overexpression on c-Myc expression and the abilities of cell proliferation, migration and invasion in curcumin-treated cells (Fig. S2A-D). These results suggested that the miR-192-5p/c-Myc axis may mediate the anticancer effects of curcumin in NSCLC.

Curcumin inactivates the Wnt/ $\beta$-catenin signaling pathway by upregulating miR-192-5p expression in NSCLC cells in vitro. To determine whether curcumin affected the Wnt/ $\beta$-catenin signaling pathway through miR-192-5p, the expression of Wnt/ $\beta$-catenin signaling pathway-related proteins was detected in A427 and A549 cells transfected with in-miR-192-5p followed by treatment with $20 \mu \mathrm{M}$ curcumin for $48 \mathrm{~h}$ using western blotting. The expression levels of $\beta$-catenin, cyclin D1 and c-Myc were significantly reduced by curcumin treatment alone compared with the control group (Fig. 6A and B); however, these decreased expression levels were subsequently significantly reversed in cells treated with curcumin and transfected with in-miR-192-5p compared with the curcumin + in-miR-NC group. These findings suggested that the curcumin-induced inactivation of the Wnt/ $\beta$-catenin signaling pathway in NSCLC may depend on the high expression levels of miR-192-5p. 
A

hsa-miR-192-5p

3' CCGACAgUUAAGUAUCCAGUC 5'

c-Myc-3'UTR-WT

5'...CAAAGGAGAUACAAGAGGUCAAA....3'

c-Myc-3'UTR-MUT

5'...CAAAGGAGAUACAAGUCCAGUAA....3'

B

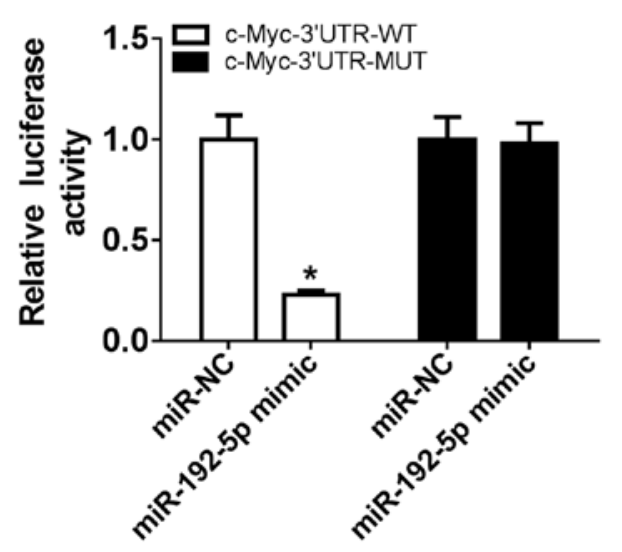

D

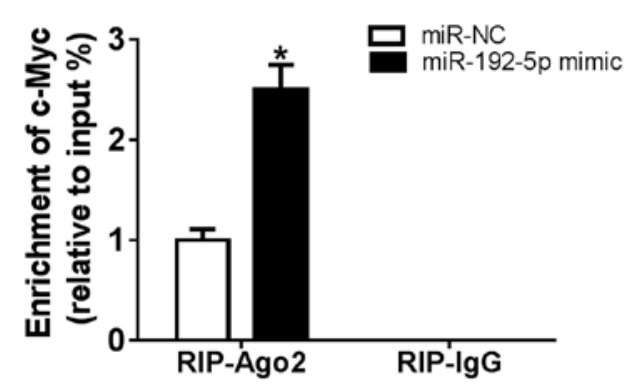

C

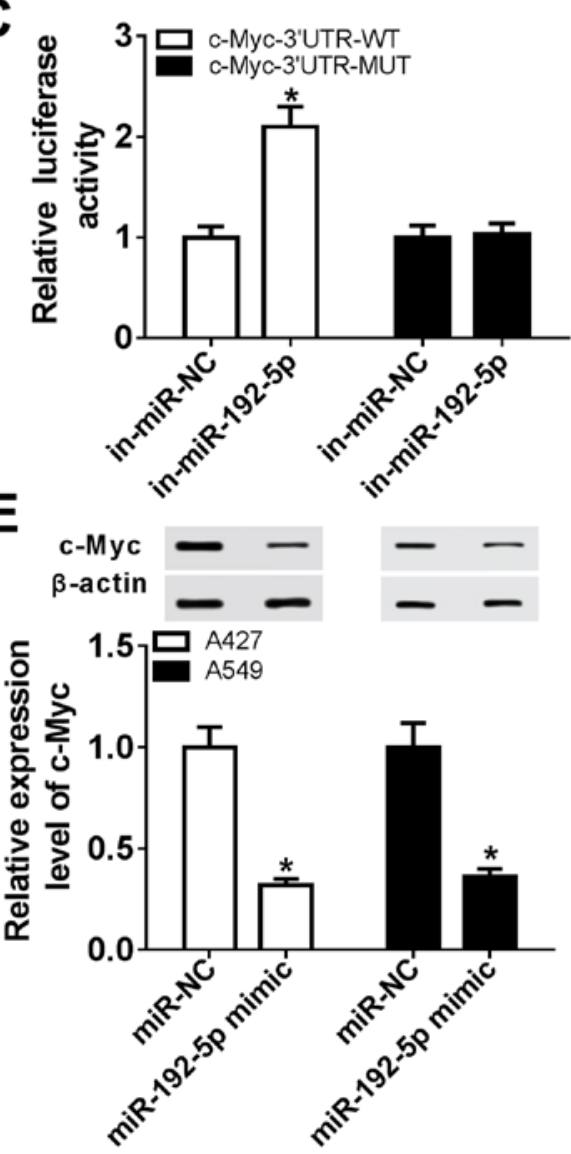

Figure 4. Experimental validation of c-Myc as a target gene of miR-192-5p. (A) Putative target binding site (red) of miR-192-5p in c-Myc was determined using computational predictions on the TargetScanHuman database. (B and C) Dual-luciferase reporter assay was used to determine the relative luciferase activities of the c-Myc-3'UTR-WT/MUT in 293T cells following the co-transfection with the (B) miR-192-5p mimic or miR-NC or (C) in-miR-192-5p or in-miR-NC. (D) RNA immunoprecipitation assay was performed to further identify c-Myc expression levels in $293 \mathrm{~T}$ cells transfected with miR-192-5p mimic/NC. (E) Western blotting was used to analyze the effect of miR-192-5p expression on the expression levels of c-Myc in A427 and A549 cells. * $<<0.05$ vs. miR-NC or in-miR-NC. miR, microRNA; UTR, untranslated region; WT, wild-type; MUT, mutant; NC, negative control; in, inhibitor; Ago2, argonaute 2.

\section{Discussion}

Curcumin, isolated from the rhizomes of the plant Curcuma longa, has been reported to exhibit various anti-inflammatory, antiangiogenic, antiproliferative and antioxidant properties $(29,30)$. It has been suggested that curcumin may induce apoptosis in malignant cells, and therefore, it demonstrates great potential as a cancer treatment (31). In addition, several studies have observed that curcumin exerts growth suppressive effects in both small cell lung cancer and NSCLC cells $(32,33)$; however, the effect of curcumin in NSCLC, in addition to its associated mechanism of action, remain poorly understood. The present study confirmed the cytotoxicity of curcumin in human NSCLC cell lines A427 and A549, and curcumin was found to inhibit cell proliferation, migration and invasion in a dose-dependent manner. Moreover, the expression levels of miR-192-5p and c-Myc were discovered to be increased and decreased, respectively, following curcumin exposure, which suggested that miR-192-5p upregulation may be an important event for the curcumin-mediated antiproliferative, antimigratory and anti-invasive effects.

The close interaction between miRNAs and the anti-lung cancer role of curcumin has been previously reported. For example, Zhang et al $(34,35)$ demonstrated that curcumin promoted apoptosis in cisplatin-resistant A549 cells through miRNA signaling pathways, including the downregulation of miR-186 expression. Zhan et al (36) constructed a miRNA gene network that is attributed to the antimetastatic role of curcumin in A549 cells, such as let-7a-3p, miR-1262 and miR-330-5p. Similarly, Jiao et al (37) identified that curcumin induces metastasis inhibition depending on a miRNA/transcription factor/target gene network, including the $\mathrm{miR}-34 \mathrm{a}-5 \mathrm{p} / \mathrm{miR}-34 \mathrm{c}-5 \mathrm{p} / \mathrm{miR}-302 \mathrm{~b}-3 \mathrm{p} / 1 \mathrm{ymphoid}$ enhancer-binding factor 1/cyclin D1/Wnt1/c-Myc axis. Moreover, curcumin has also been found to be effective in increasing paclitaxel sensitivity in cancer stem cells, which may occur by interacting with miRNAs $(38,39)$. In the present 


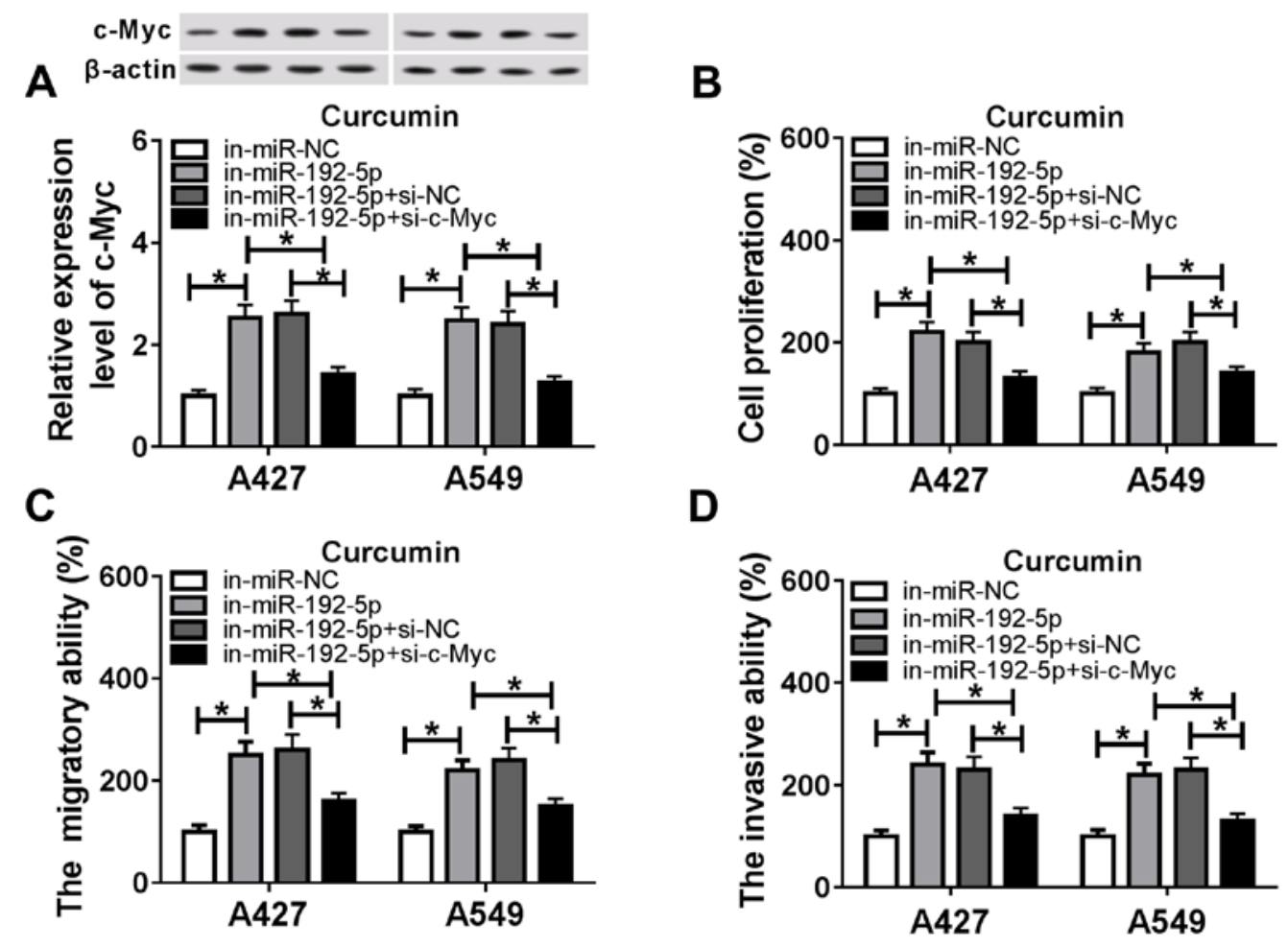

Figure 5. Effect of c-Myc genetic knockdown on the curcumin/miR-192-5p axis in non-small-cell lung cancer progression. A427 and A549 cells were transfected with in-miR-192-5p or in-miR-NC and co-transfected with si-c-Myc or si-NC. (A) Expression levels of c-Myc were determined in A427 and A549 cells using reverse transcription-quantitative PCR. (B) Cell proliferation in A427 and A549 cells using a Cell Counting Kit-8 assay. Rate of (C) cell migration and (D) invasion in A427 and A549 cells were determined using a Transwell assay or Matrigel assay, respectively. "P<0.05. miR, microRNA; in, inhibitor; NC, negative control; si, small interfering RNA.
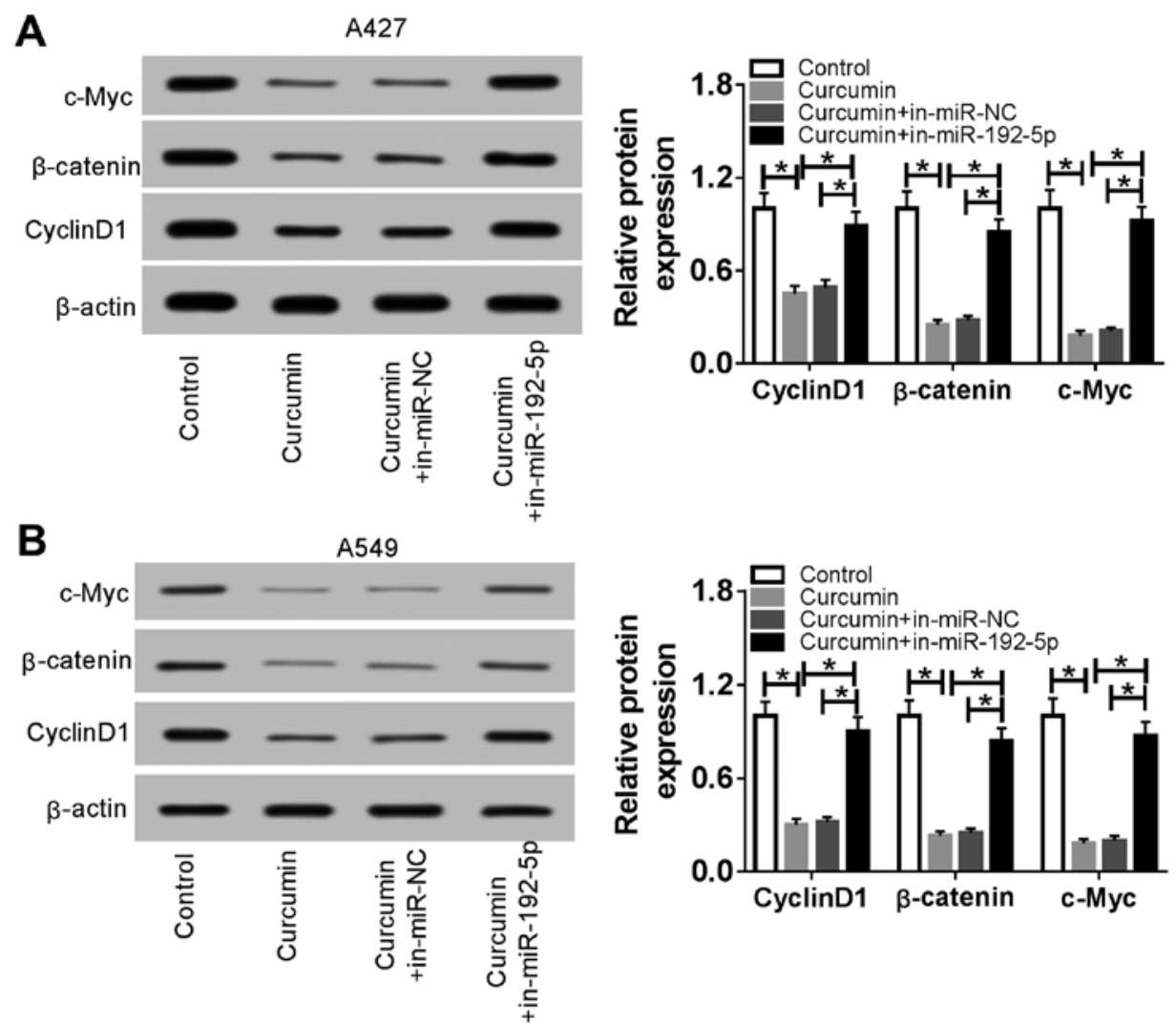

Figure 6. Role of miR-192-5p in the Wnt/ $\beta$-catenin signaling pathway in non-small-cell lung cancer following curcumin treatment. A427 and A549 cells were transfected with in-miR-192-5p and the effect of miR-192-5p knockdown on the Wnt/ $/$-catenin signaling pathway-related proteins were determined following $20 \mu \mathrm{M}$ curcumin treatment. Expression levels of $\beta$-catenin, cyclin D1 and c-Myc were detected in (A) A427 and (B) A549 cells using western blotting. "P<0.05. miR, microRNA; in, inhibitor; NC, negative control. 
study, the increased expression levels of miR-192-5p initiated the antiproliferative, antimigratory and anti-invasive roles of curcumin in A549 and A427 cells by targeting c-Myc expression. Taken together, these results suggested that curcumin could be an epigenetic agent that could provide a new therapeutic strategy for cancer treatment, and the miRNAs/genes network, including the miR-192-5p/c-Myc axis, may serve a vital role in the anticancer activity of curcumin in lung cancer.

Previous studies have shown that the increased expression levels of miR-192-5p contribute to curcumin-induced antitumor effects in NSCLC $(13,14)$. To date, the tumor-suppressive activities of curcumin have mainly focused on its ability to promote cell apoptosis, and enhance chemo- and radiosensitization, in addition to its ability to inhibit cell proliferation and migration in lung cancer cells, and modulate miRNAs $(11,40)$. For example, antagonizing miR-192-5p was found to attenuate curcumin-stimulated apoptosis promotion in H460, A427 and A549 cells through the p53/miR-192-5p/X-linked inhibitor of apoptosis protein axis (13), of which miR-192-5p was identified as one of the most responsive miRNAs following curcumin treatment. Another study reported that the ectopic expression of miR-192-5p enhanced the effects of curcumin on the inhibition of cell viability and the promotion of apoptosis in A549 cells through the PI3K/AKT signaling pathway (14). Nonetheless, to the best of our knowledge, numerous roles of miR-192-5p exist in NSCLC progression, which have not been investigated in detail, such as migration and invasion. The present study suggested that the upregulation of miR-192-5p contributed to the curcumin-induced antiproliferative effects in both A549 and A427 cells. Furthermore, the migratory and invasive abilities of the cells were also significantly inhibited by curcumin treatment, whereas miR-192-5p inhibition could partially reverse the curcumin-induced antimigration and anti-invasion effects through inactivation of the $\mathrm{Wnt} / \beta$-catenin signaling pathway by decreasing the expression levels of $\beta$-catenin, cyclin D1 and c-Myc.

As a well-documented oncogene, c-Myc has been known to be extensively involved in cell cycle progression and apoptosis (41). The dysregulation of this gene has been associated with multiple types of cancer, including NSCLC (42): For example, it was reported that c-Myc was a major target for miR-145, which negatively mediated eukaryotic translation initiation factor $4 \mathrm{E}$, a downstream target of c-Myc, in NSCLC (25); epidermal growth factor receptor was discovered to promote lung tumorigenesis through activating c-Myc, which in turn stimulated miR-7 expression (43); and increased expression levels of c-Myc were demonstrated to reverse the inhibitory effect of miR-449c on NSCLC tumor growth in vivo (44). In addition, cell proliferation and invasion in A549 cells were also altered by the miR-376a/c-Myc axis in a previous study (45). Therefore, to investigate whether c-Myc was a direct target gene of miR-192-5p, a dual-luciferase reporter assay and RIP were performed; it was observed that c-Myc was negatively regulated by miR-192-5p, and that the overexpression of c-Myc relieved the inhibition of miR-192-5p on A427 and A549 cell proliferation, migration and invasion. However, the anticancer effect of miR-192-5p induction through c-Myc knockdown was only investigated in the human NSCLC cell lines, A427 and A549, in the present study, and to the best of our knowledge, there is currently no published xenograft experiments that have focused on the increased expression levels of miR-192-5p as the underlying mechanism of the curcumin-mediated tumor-suppressive role $(11,13,14)$.

The Wnt pathway, especially the Wnt/ $\beta$-catenin dependent pathway is the main target for therapeutic interventions in cancer types, including lung cancer $(46,47)$. Activating the $\mathrm{Wnt} / \beta$-catenin leads to the accumulation of $\beta$-catenin and further promotes oncogenes, including c-Myc and Cyclin D1 (48). Curcumin can inhibit the Wnt/ $\beta$-catenin pathway in lung cancer stem cells and lung cancer cells $(15,16)$. Moreover, miR-192-5p is also associated with the Wnt//-catenin pathway in malignant tumors $(49,50)$. However, the relationship between curcumin, miR-192-5p and the Wnt/ $\beta$-catenin pathway have not been previously investigated. Thus, the present results suggested that miR-192-5p upregulation mediated the antitumor role of curcumin in NSCLC by activating the Wnt//-catenin pathway and targeting c-Myc.

In conclusion, the present study demonstrated that curcumin exhibited antiproliferative and antimigratory and anti-invasive properties in NSCLC in vitro, which were largely mediated through the increased expression levels of miR-192-5p by targeting c-Myc and inhibiting the Wnt/ $\beta$-catenin signaling pathway. These findings suggested a novel mechanism for the anticancer function of curcumin and suggested that the restoration of miR-192-5p could be used as a novel therapeutic approach for NSCLC treatment.

\section{Acknowledgements}

Not applicable.

\section{Funding}

No funding was received.

\section{Availability of data and materials}

The datasets used and/or analyzed during the current study are available from the corresponding author on reasonable request.

\section{Authors' contributions}

YP and CZ conceptualized and designed the experiments, analyzed the data, and prepared the original draft of the manuscript. YS and ZL performed the experiments, collected and validated the data, and revised it critically for important intellectual content prior to reviewing and editing. All authors read and approved the final manuscript.

\section{Ethics approval and consent to participate}

Not applicable.

\section{Patient consent for publication}

Not applicable.

\section{Competing interests}

The authors declare that they have no competing interests. 


\section{References}

1. Gerber DE, Laccetti AL, Xuan L, Halm EA and Pruitt SL: Impact of prior cancer on eligibility for lung cancer clinical trials. J Natl Cancer Inst 106: pii: dju302, 2014.

2. Bray F, Ferlay J, Soerjomataram I, Siegel RL, Torre LA and Jemal A: Global cancer statistics 2018: GLOBOCAN estimates of incidence and mortality worldwide for 36 cancers in 185 countries. CA Cancer J Clin 68: 394-424, 2018.

3. Lochowski M, Lochowska B, Rebowski M, Brzezinski D, Cieslik-Wolski B and Kozak J: Five-year survival analysis and prognostic factors in patients operated on for non-small cell lung cancer with N2 disease. J Thorac Dis 10: 3180-3186, 2018.

4. Stinchcombe TE and Socinski MA: Current treatments for advanced stage non-small cell lung cancer. Proc Am Thorac Soc 6: 233-241, 2009.

5. Correction: New perspectives of curcumin in cancer prevention. Cancer Prev Res (Phila) 10: 371, 2017.

6. Ibrahim S, Tagami T, Kishi T and Ozeki T: Curcumin marinosomes as promising nano-drug delivery system for lung cancer. Int J Pharm 540: 40-49, 2018.

7. Maran A, Yaszemski MJ, Kohut A and Voronov A: Curcumin and osteosarcoma: Can invertible polymeric micelles help? Materials (Basel) 9: pii: E520, 2016.

8. Khan MA, Akhtar N, Sharma V and Pathak K: Product development studies on sonocrystallized curcumin for the treatment of gastric cancer. Pharmaceutics 7: 43-63, 2015.

9. Ye MX, Li Y, Yin H and Zhang J: Curcumin: Updated molecular mechanisms and intervention targets in human lung cancer. Int $\mathrm{J}$ Mol Sci 13: 3959-3978, 2012.

10. Du X, Zhang J, Wang J, Lin X and Ding F: Role of miRNA in lung cancer-potential biomarkers and therapies. Curr Pharm Des 23: 5997-6010, 2018.

11. Lelli D, Pedone C, Majeed M and Sahebkar A: Curcumin and lung cancer: The role of microRNAs. Curr Pharm Des 23: 3440-3444, 2017.

12. Simental-Mendia LE and Sahebkar A: Modulation of microRNAs by curcumin in pancreatic cancer. Clin Nutr 35 $1585,2016$.

13. Ye M, Zhang J, Zhang J, Miao Q, Yao L and Zhang J: Curcumin promotes apoptosis by activating the p53-miR-192-5p/215-XIAP pathway in non-small cell lung cancer. Cancer Lett 357: 196-205, 2015.

14. Jin H, Qiao F, Wang Y, Xu Y and Shang Y: Curcumin inhibits cell proliferation and induces apoptosis of human non-small cell lung cancer cells through the upregulation of miR-192-5p and suppression of PI3K/Akt signaling pathway. Oncol Rep 34: 2782-2789, 2015

15. Lu Y, Wei C and Xi Z: Curcumin suppresses proliferation and invasion in non-small cell lung cancer by modulation of MTA1-mediated Wnt/ $\beta$-catenin pathway. In Vitro Cell Dev Biol Anim 50: 840-850, 2014.

16. Zhu JY, Yang X, Chen Y, Jiang Y, Wang SJ, Li Y, Wang XQ, Meng Y,Zhu MM, Ma X, et al: Curcumin suppresses lung cancer stem cells via inhibiting wnt/ $\beta$-catenin and sonic hedgehog pathways. Phytother Res 31: 680-688, 2017.

17. He M, Li Y, Zhang L, Li L, Shen Y, Lin L, Zheng W, Chen L, Bian X, Ng HK and Tang L: Curcumin suppresses cell proliferation through inhibition of the Wnt/ $\beta$-catenin signaling pathway in medulloblastoma. Oncol Rep 32: 173-180, 2014.

18. Dou H, Shen R, Tao J, Huang L, Shi H, Chen H, Wang Y and Wang T: Curcumin suppresses the colon cancer proliferation by inhibiting wnt $/ \beta$-catenin pathways via miR-130a. Front Pharmacol 8: 877, 2017.

19. Wang JY, Wang X, Wang XJ, Zheng BZ, Wang Y, Wang X and Liang B: Curcumin inhibits the growth via Wnt/ $\beta$-catenin pathway in non-small-cell lung cancer cells. Eur Rev Med Pharmacol Sci 22: 7492-7499, 2018.

20. Xu JH, Yang HP, Zhou XD, Wang HJ, Gong L and Tang CL: Role of Wnt inhibitory factor-1 in inhibition of bisdemethoxycurcumin mediated epithelial-to-mesenchymal transition in highly metastatic lung cancer 95D cells. Chin Med J (Engl) 128: 1376-1383, 2015.

21. Zhang X, Peng Y, Huang Y, Yang M, Yan R, Zhao Y, Cheng Y, Liu X, Deng S, Feng X, et al: SMG-1 inhibition by miR-192/-215 causes epithelial-mesenchymal transition in gastric carcinogenesis via activation of Wnt signaling. Cancer Med 7: 146-156, 2018.
22. Chen J, Chen Z, Huang J, Chen F, Ye W, Ding G and Wang X: Bioinformatics identification of dysregulated microRNAs in triple negative breast cancer based on microRNA expression profiling. Oncol Lett 15: 3017-3023, 2018

23. Zhang H, Zhang C, Feng R, Zhang H, Gao M and Ye L: Investigating the microRNA-mRNA regulatory network in acute myeloid leukemia. Oncol Lett 14: 3981-3988, 2017

24. Zhang T, Chen Y, Ge Y, Hu Y, Li M and Jin Y: Inhalation treatment of primary lung cancer using liposomal curcumin dry powder inhalers. Acta Pharm Sin B 8: 440-448, 2018.

25. Livak KJ and Schmittgen TD: Analysis of relative gene expression data using real-time quantitative PCR and the 2(-Delta Delta C(T)) method. Methods 25: 402-408, 2001.

26. Kang HW, Crawford M, Fabbri M, Nuovo G, Garofalo M, Nana-Sinkam SP and Friedman A: A mathematical model for microRNA in lung cancer. PLoS One 8: e53663, 2013.

27. Cui J, Mo J, Luo M, Yu Q, Zhou S, Li T, Zhang Y and Luo W: C-Myc-activated long non-coding RNA H19 downregulates miR-107 and promotes cell cycle progression of non-small cell lung cancer. Int J Clin Exp Pathol 8: 12400-12409, 2015.

28. Chen Z, Zeng H, Guo Y, Liu P, Pan H, Deng A and Hu J: MiRNA-145 inhibits non-small cell lung cancer cell proliferation by targeting c-Myc. J Exp Clin Cancer Res 29: 151, 2010.

29. Park W, Amin AR, Chen ZG and Shin DM: New perspectives of curcumin in cancer prevention. Cancer Prev Res (Phila) 6: 387-400, 2013.

30. Gupta SC, Patchva S and Aggarwal BB: Therapeutic roles of curcumin: Lessons learned from clinical trials. AAPS J 15: 195-218, 2013.

31. Reuter S, Eifes S, Dicato M, Aggarwal BB and Diederich M: Modulation of anti-apoptotic and survival pathways by curcumin as a strategy to induce apoptosis in cancer cells. Biochem Pharmacol 76: 1340-1351, 2008.

32. Datta R, Halder SK and Zhang B: Role of TGF- $\beta$ signaling in curcumin-mediated inhibition of tumorigenicity of human lung cancer cells. J Cancer Res Clin Oncol 139: 563-572, 2013.

33. Yang CL, Liu YY, Ma YG, Xue YX, Liu DG, Ren Y, Liu XB, Li Y and Li Z: Curcumin blocks small cell lung cancer cells migration, invasion, angiogenesis, cell cycle and neoplasia through Janus kinase-STAT3 signalling pathway. PLoS One 7: e37960, 2012.

34. Zhang J, Zhang T, Ti X, Shi J, Wu C, Ren X and Yin $\mathrm{H}$ : Curcumin promotes apoptosis in A549/DDP multidrug-resistant human lung adenocarcinoma cells through an miRNA signaling pathway. Biochem Biophys Res Commun 399: 1-6, 2010.

35. Zhang J, Du Y, Wu C, Ren X, Ti X, Shi J, Zhao F and Yin H: Curcumin promotes apoptosis in human lung adenocarcinoma cells through miR-186* signaling pathway. Oncol Rep 24: 1217-1223, 2010.

36. Zhan JW, Jiao DM, Wang Y, Song J, Wu JH, Wu LJ, Chen QY and Ma SL: Integrated microRNA and gene expression profiling reveals the crucial miRNAs in curcumin anti-lung cancer cell invasion. Thorac Cancer 8: 461-470, 2017.

37. Jiao DM, Yan L, Wang LS, Hu HZ, Tang XL, Chen J, Wang J, Li Y and Chen QY: Exploration of inhibitory mechanisms of curcumin in lung cancer metastasis using a miRNA-transcription factor-target gene network. PLoS One 12: e0172470, 2017.

38. Lu Y, Wang J, Liu L, Yu L, Zhao N, Zhou X and Lu X: Curcumin increases the sensitivity of Paclitaxel-resistant NSCLC cells to Paclitaxel through microRNA-30c-mediated MTA1 reduction. Tumour Biol 39: 1010428317698353, 2017.

39. Suresh R, Ali S, Ahmad A, Philip PA and Sarkar FH: The role of cancer stem cells in recurrent and drug-resistant lung cancer. Adv Exp Med Biol 890: 57-74, 2016.

40. Zhang W, Bai W and Zhang W: MiR-21 suppresses the anticancer activities of curcumin by targeting PTEN gene in human non-small cell lung cancer A549 cells. Clin Transl Oncol 16: 708-713, 2014.

41. Chen S, Gu T, Lu Z, Qiu L, Xiao G, Zhu X, Li F, Yu H, Li G and Liu H: Roles of MYC-targeting long non-coding RNA MINCR in cell cycle regulation and apoptosis in non-small cell lung Cancer. Respir Res 20: 202, 2019.

42. Wang J, Jia Y, Zhao S, Zhang X, Wang X, Han X, Wang Y, Ma M, Shi J and Liu L: BIN1 reverses PD-L1-mediated immune escape by inactivating the c-MYC and EGFR/MAPK signaling pathways in non-small cell lung cancer. Oncogene 36: 6235-6243, 2017. 
43. Chou YT, Lin HH, Lien YC, Wang YH, Hong CF, Kao YR, Lin SC, Chang YC, Lin SY, Chen SJ, et al: EGFR promotes lung tumorigenesis by activating miR-7 through a Ras/ERK/Myc pathway that targets the Ets2 transcriptional repressor ERF. Cancer Res 70: 8822-8831, 2010.

44. Miao LJ, Huang SF, Sun ZT, Gao ZY, Zhang RX, Liu Y and Wang J: MiR-449c targets c-Myc and inhibits NSCLC cell progression. FEBS Lett 587: 1359-1365, 2013.

45. Wang Y, Cong W, Wu G, Ju X, Li Z, Duan X, Wang X and Gao H: MiR-376a suppresses the proliferation and invasion of non-small-cell lung cancer by targeting c-Myc. Cell Biol Int 42: 25-33, 2018.

46. Krishnamurthy Nand Kurzrock R: Targeting the Wnt/beta-catenin pathway in cancer: Update on effectors and inhibitors. Cancer Treat Rev 62: 50-60, 2018.
47. Stewart DJ: Wnt signaling pathway in non-small cell lung cancer. J Natl Cancer Inst 106: djt356, 2014.

48. Shang S, Hua F and $\mathrm{Hu} Z \mathrm{ZW}$ : The regulation of $\beta$-catenin activity and function in cancer: Therapeutic opportunities. Oncotarget 8: 33972-33989, 2017.

49. Park M, Kim M, Hwang D, Park M, Kim WK, Kim SK, Shin J, Park ES, Kang CM, Paik YK and Kim H: Characterization of gene expression and activated signaling pathways in solid-pseudopapillary neoplasm of pancreas. Mod Pathol 27: 580-593, 2014.

50. Kang DW, Lee SW, Hwang WC, Lee BH, Choi YS, Suh YA, Choi KY and Min DS: Phospholipase D1 Acts through Akt/TopBP1 and RB1 to regulate the E2F1-dependent apoptotic program in cancer cells. Cancer Res 77: 142-152, 2017. 